Article

\title{
An Acoustic Sensing Gesture Recognition System Design Based on a Hidden Markov Model
}

\author{
Bruna Salles Moreira *, Angelo Perkusich $\$ and Saulo O. D. Luiz \\ Embedded Systems and Pervasive Computing Laboratory, Electrical Engineering Department, \\ Federal University of Campina Grande, Campina Grande, Paraíba 58429-900, Brazil; \\ perkusic@embedded.ufcg.edu.br (A.P.); saulo@dee.ufcg.edu.br (S.O.D.L.) \\ * Correspondence: bruna.moreira@ee.ufcg.edu.br
}

Received: 7 July 2020; Accepted: 24 August 2020; Published: 26 August 2020

check for updates

\begin{abstract}
Many human activities are tactile. Recognizing how a person touches an object or a surface surrounding them is an active area of research and it has generated keen interest within the interactive surface community. In this paper, we compare two machine learning techniques, namely Artificial Neural Network (ANN) and Hidden Markov Models (HMM), as they are some of the most common techniques with low computational cost used to classify an acoustic-based input. We employ a small and low-cost hardware design composed of a microphone, a stethoscope, a conditioning circuit, and a microcontroller. Together with an appropriate surface, we integrated these components into a passive gesture recognition input system for experimental evaluation. To perform the evaluation, we acquire the signals using a small microphone and send it through the microcontroller to MATLAB's toolboxes to implement and evaluate the ANN and HMM models. We also present the hardware and software implementation and discuss the advantages and limitations of these techniques in gesture recognition while using a simple alphabet of three geometrical figures: circle, square, and triangle. The results validate the robustness of the HMM technique that achieved a success rate of $90 \%$, with a shorter training time than the ANN.
\end{abstract}

Keywords: gesture recognition; acoustic-based input; artificial neural network; Hidden Markov models

\section{Introduction}

Day-to-day activities are a combination of tactile and auditory sensations. We play, listen, and interact with a variety of objects every day. Some of these objects are recording our activities, such as the touch screens of smartphones or tablets. Thus, adding sensory perception to objects and surfaces that surround us daily is an active area of research, and it has generated keen interest within the interactive surface community [1-4].

By connecting a microphone to a surface, a signal is acquired by an acoustic sensor, and it is then possible to analyze the acquired signal to identify information for the development of tangible acoustic interfaces [5]. These interfaces work based on the principle that, when an object is touched and manipulated, the vibrations it produces can be acquired and analyzed to infer how interaction with the object occurs [6].

Acoustic sensing has been explored as a powerful tool to retrieve information from a user's interaction with a surface or object. Some of this information can be used by developers to enhance interfaces with new features, or, most importantly, to improve interaction on current interfaces [6], which can provide more expression to user-defined gestures and expand the interaction input language.

In contrast to camera-based gesture recognition systems, all sensor elements of the acoustic detection technique can be integrated into the surface, and this method does not suffer from lighting and occlusion problems [7]. 
Researchers have explored several acoustic-based input techniques. These techniques are classified either as passive or active approaches. Passive approaches detect a user's input, acquiring and analyzing sounds generated by the user's actions, without the need for an active component, only depending on the properties of the object used (its structure, material, or exclusive properties). Besides, for active approaches, an actuator must be used to interact with a surface, such as a loudspeaker.

Acoustic detection has been the subject of intense research in recent decades. Various algorithms for processing acoustic information have been explored. These include Artificial Neural Networks [8,9], Convolution Neural Networks (CNN) [10], Support Vector Machine (SVM) [11,12], Decision Trees [13], and Hidden Markov Models [14]. These efforts led to the development of systems used in various acoustic tasks, including gesture and textures recognition, the spatial location of the surface touch, and object classification.

In the context of this work, our focus is to answer the following Research Questions $(R Q)$ :

- $\quad R Q 1$ : What are the tools, applications, and trends involving these machine learning classifiers?

- $\quad R Q 2$ : What are the signal characteristics when using a machine learning technique to recognize acoustic patterns?

- RQ3: What is the most suitable machine learning technique for acoustic gesture recognition applications?

We distinguish from previous work by implementing acoustic-based input in gesture recognition applications comparing two different techniques: Artificial Neural Network (ANN) and Hidden Markov Models (HMM). First, we present a low-cost hardware design that is composed of a microphone, a stethoscope, a conditioning circuit, and a microcontroller. Subsequently, we integrated these components with a surface to implement a passive gesture recognition system. Second, we evaluated the dataset acquired for developing and training the ANN and HMM models. For ANNs, we investigate algorithms to process the signal to achieve higher accuracy rates due to the low success rate obtained using unprocessed signals. Finally, we evaluated the HNN model. The results validate the robustness of the HMM technique that achieved a success rate of $90 \%$, with a shorter training time than the ANN. We also discuss how to generate code from the trained HMM, cross-compile it automatically, and embed it into low-cost hardware. Thus, after training, the system would be independent of MATLAB software, which has a higher cost.

The rest of the paper is organized, as follows. In Section 2, we introduce a review of the related works, while in Section 3 we present the materials and methods. In Section 4, we present the experiments and, in Section 5, the discussion of the results against the defined research questions. Finally, in Section 6 we present the conclusions and future work.

\section{Acoustic Sensing Related Works}

In this section, we present a review of scientific papers that use the acoustic detection technique, focusing on the three research questions defined in the introduction.

Nine keywords were defined in order to search for papers aiming to answer the research question enumerated in the introduction, and are as follows:

\section{Acoustic Sensing, Scratch Input, Acoustic Tracking, Tactile Input, Gesture Classification, Sound based classification, Sound Sensing, Acoustic-based input, and Fingertip Gesture.}

Besides, we defined two general selection criteria: (i) the papers were peer-reviewed and (ii) English was the language used to write the paper.

Scratch Input [13] is an acoustic input technique that is based on the sound originated when a nail is dragged over the surface of textured material. Harrison and Hudson [13] used a microphone attached to a modified stethoscope, particularly suitable for amplifying sound and detecting high-frequency noise. A microphone converts the sound into an electrical signal, which is then amplified and acquired 
by a computer using an audio input connector. This hardware can be effortlessly attached to existing surfaces, turning them into large, non-powered, ad hoc entry surfaces.

The acoustic pattern recognizer proposed by Harrison and Hudson [13] employs a decision tree based on peak count and amplitude variation. A more sophisticated recognition mechanism can incorporate other dimensions, e.g., frequency and duration, and it may support a broader set of gestures and higher precision. Subsequently, other works started to use more advanced techniques, such as Hambone [14] with HMM, Stane [8] with ANN, and TapSense [12] with SVM.

Hambone [14] is a light and discreet system that offers quick access and the ability to multitask for the interaction of mobile devices. Hambone [14] uses two small piezoelectric sensors placed on the wrist or ankle of the user. When a user moves either the hands or feet, the sounds generated voyage to the device via bone conduction. Subsequently, it transmits the signals digitally to a mobile device or computer for processing using HMMs. Afterwards, the signals are mapped to a set of commands that control applications.

Two limitations were found in the current implementation of Hambone [14]. Whenever a user makes a gesture, there is a two-second lag from the initiation of the gesture to have the final command issued and, when training the system to recognize more than four gestures, the HMM training sessions become time-consuming.

Stane [8] is a small device with an embedded microphone and several textures projected onto its surface. The device classifies the sounds produced when rubbing different areas. Stane [8] uses a two-stage classification process, with instantaneous low-level classification and higher-level classifiers that aggregate the evidence of the first stage over time. For instant classification, the input audio is classified by Multi-Layer Perceptron (MLP). However, Stane [8] needs a textured device case and it cannot be used on any surface, differently of what we are proposing in this paper: a passive acoustic-based input classification easily incorporated into a surface.

TapSense [12] uses a microphone connected to an interactive surface to differentiate between touches of different objects and parts of the finger, and it uses the SVM classifier. Nevertheless, the following amounts of data points were collected per participant to train this classifier: 200 data points for the mobile input set, 160 data points for the table finger set, and 280 data points for the table toolset. It has been identified that the user boredom and fatigue became problematic in more extended study durations.

Most recently, we can find papers using machine learning techniques with high computational costs, such as a combination of motion and audio features to train the neural network, such as Pentelligence [9], or even using Convolutional Neural Network, such as Mingshi et al. [10]. Indeed, it is required massive amounts of data, millions of samples.

For example, Pentelligence [9] is based on a pen for handwritten digit recognition. It can be used on regular paper, and no separate dedicated tracking device is required. The authors of Pentelligence [9] found that combining the strengths of audio and motion features produces better results when motion data are classified first. Neural networks trained on the sound emissions of the pen tip are used for evaluation purposes. However, the authors of Pentelligence [9] have used a contact sensor, a microphone, an accelerometer, and a gyroscope in order to achieve a reasonable recognition rate. All of these sensors and the high computational cost became problematic in low-cost applications.

Chen et al. [10] explore the possibility of extending the input and interactions beyond the screen of the mobile device by using ad hoc adjacent surfaces. The proposed system Ipanel uses the acoustic signals from the fingers' sliding on the table for tracking. Different features are extracted by exploiting the spatio-temporal and frequency domain properties of the produced acoustic signals. The features are transformed into images, and then Chen et al. [10] employed CNN to recognize the finger movement on the table. Ipanel can support not only commonly used gestures (such as click, flip, scroll, and zoom) recognition and handwriting (10 numbers and 26 letters) recognition at high accuracy.

Further, Ipanel's performance is robust against different levels of ambient noise and different surface materials. Although, the recognition accuracy significantly decreases when the fingers are 
$20 \mathrm{~cm}$ away from the mobile, mainly because the signals become too weak to be used for recognition, and the five-layer $\mathrm{CNN}$ adopted has a high computational cost.

\section{Materials and Methods}

We conducted an experiment focusing on the dataset input for the classifiers in the gesture classification problem to improve the analysis. Our goal is to have simple hardware and low computational cost for the machine learning algorithm that can achieve high success rate with at most ten drawings per gesture and short training time (maximum two minutes per gesture), wherefore the user is not fatigued in the training stage. Such a goal is similar to the one that motivated the authors of TapSense [12].

Based on a previous study, several papers focus on gesture recognition using a subset of machine learning techniques, but some of the most common techniques with low computational cost are ANN and HMM. Therefore, the next section presents our platform, analyzes these two machine learning techniques, and compares them.

\section{Platform}

Figure 1 illustrates the operation of the proposed system. When a nail (A) scratches a surface, it produces a series of mechanical vibrations, which propagate through the surface and they are acquired by the microphone (C), which is connected to the stethoscope cannula (B). The use of stethoscopes to acquire sounds naturally provides a high level of suppression of environmental noise. The microphone converts the sound into an electrical signal. By covering the microphone with the stethoscope, the first may pick up a less noisy signal from the environment. This allows for impacts to be promptly segmented from any background noise with a simple amplitude threshold [15]. The microphone signal is then filtered and amplified by a conditioning circuit (D), and acquired by an analog to digital converter of a microcontroller. Then the microcontroller sends the digital signal via serial communication (UART) to the computer (E), which finally will be capable of recognizing the gesture performed $(\mathrm{F})$ on the surface by one of the machine learning applications developed in MATLAB.

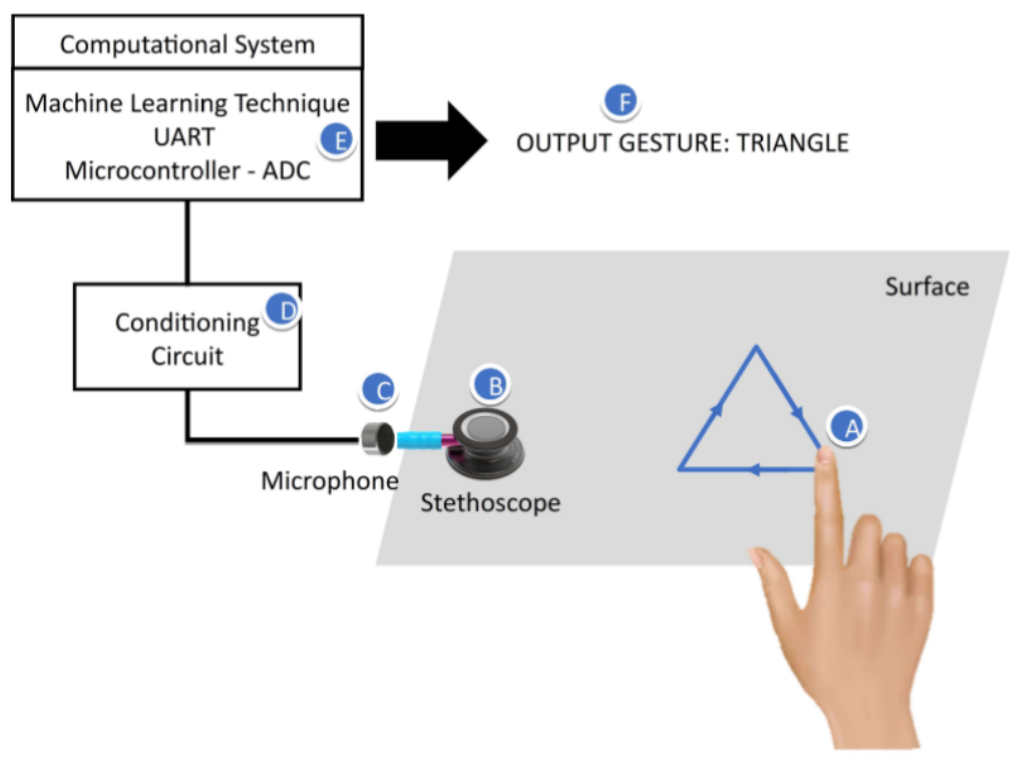

Figure 1. Functioning of the proposed system.

The stethoscope has a dual lumen design. The mini electret microphone dimensions are $0.16 \times 0.06$ inches, and it has to be small enough to fit inside the stethoscope cannula. The conditioning circuit is a low-cost microphone amplifier by Adafruit MAX4466. This circuit has a trimmer pot to 
adjust the gain from $25 \times$ to $125 \times$. The output is rail-to-rail and the supply voltage operation is $2.4 \mathrm{~V}$ to $5.5 \mathrm{~V}$.

The microcontroller used in this project is the Arduino Nano V3.0, which has, as primary characteristics, its small size and low cost, allowing greater flexibility for the use of this board in projects whose size is essential. The ADC of this microcontroller is a 10-bit, and the UART transmission rate used in this project is $115,200 \mathrm{bits} / \mathrm{s}$.

Because sound travels through solid and liquid materials much more efficiently than through the air, the surface's importance is reaffirmed. Thus, while the friction of the nail on a surface only produces an audible and smooth noise, the signal is propagated considerably better through the solid material. This superior sound propagation means that a signal is propagated over a longer distance, but it is also better-preserved [13]. As the speed of sound propagation in aluminum is among the highest among the common materials, around $6300 \mathrm{~m} / \mathrm{s}$, an aluminum surface was chosen to carry out the experiments. The stethoscope provides a high level of suppression of environmental noise to the microphone. Still, all of the experiments were performed against the maximum level of ambient noise of $45 \mathrm{~dB}$.

This work aims to compare the robustness of the HMM and the ANN techniques to recognize gestures on surfaces while using a low-cost acoustic sensing gesture recognition system concerning time and physical memory usage. Accordingly, we established the following requirements: ten drawings per gesture at the most, and short training time, a maximum of two minutes per gesture. We defined, for the initial alphabet, three geometrical figures: circle, square, and triangle. Such a small initial alphabet thus prevents time-consuming training. Hence, at the beginning of the application, the user is notified to start drawing one of those figures.

In contrast, for training the recognition of four parts of the finger (tip, pad, nail, and knuckle), Tapsense [12] requires collecting per participant 200 data points for the mobile input set, 160 data points for the table finger set, and 280 data points for the table toolset. When training Hambone [14] to recognize more than 3-4 gestures, the HMM training sessions become extremely tedious. The authors of Tapsense and Hambone found that user boredom and fatigue became problematic in a longer study duration. Furthermore, an excessive number of gestures in the dictionary may lead to high computational costs.

\section{Experiments}

This section describes experiments in order to evaluate the performance of the proposed methods and analyzes them using the machine learning techniques ANN and HMM.

\subsection{Artificial Neural Networks Experiment}

Finding an optimal setup for neural networks is challenging because many parameters have to be considered. Determining the optimal number of hidden layers and neurons for all classifiers is a crucial aspect. If the network's complexity is too low to model the dataset's characteristics, the error rates are higher. If the complexity is too high, extended training and recall times are the consequence [16].

Hence, to train the ANN, we collected ten drawings of each gesture (circle, square, and triangle). Subsequently, all of the data were concatenated to be used as input for MATLAB's Neural Net Pattern Recognition Toolbox. The configuration offered by this toolbox is a two-layer feed-forward network with sigmoid hidden and softmax output neurons. The network is trained with scaled conjugate gradient backpropagation. By setting the number of neurons in the hidden layer to 10 neurons, we had the best performance, because we have minimized the cross-entropy while constraining overfitting. If more than ten neutrons were placed in the hidden layer, the model started to overfit the data and decrease the estimation accuracy. 


\subsubsection{Dataset for ANN}

Achieving a high generalization in one neural network is challenging due to the differences that were observed in our dataset's scratching styles. The complexity of the optimal topology is possibly too high to be sufficiently trained, given our limited set of training samples. For that reason, we had to process the signal that was presented to train the neural network.

\subsubsection{Raw Time Signal}

The first dataset we have used to train the ANN was the raw time signal, as shown in Figure 2. We can see the difference between the square (Figure $2 b$ ) and the triangle (Figure 2c) due to the difference in the number of peaks related to the stops at the edges of the drawing. However for the ANN, all of these peaks representing the gesture in the surface (Figure 2) make the ANN classification more complex, because each gesture will have a completely different number and position of peaks and grooves.

Another complicating factor for the neural network is that each person can start the gesture at a different time, making it difficult for the ANN to identify this first peak and classify the signal correctly because each gesture possibly starts at a different time instant. Accordingly, with this dataset, we only had $33 \%$ of success.

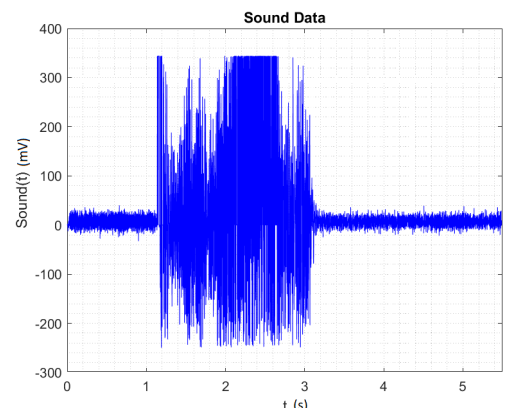

(a)

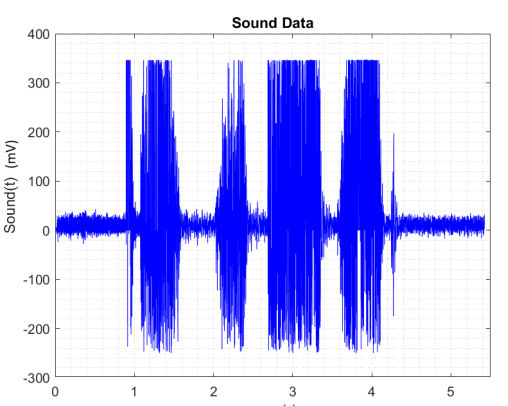

(b) ${ }^{\mathrm{t}}$

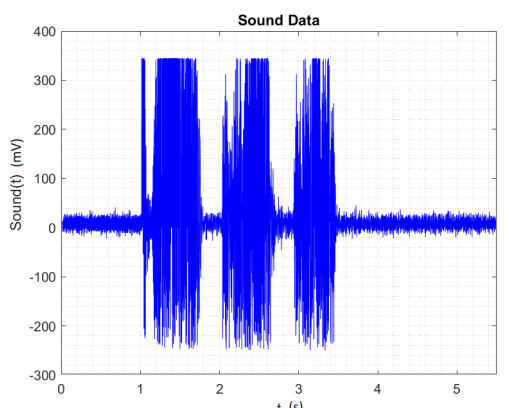

(c)

Figure 2. (a) Time signal for Circle. (b) Time signal for Square. (c) Time signal for Triangle.

\subsubsection{FFT Signal}

The second dataset attempt to train the ANN was using the Fast Fourier Transform (FFT) signal, as shown in Figure 3, but it was even harder for the ANN to make the recognition and we had a success rate of $15 \%$.

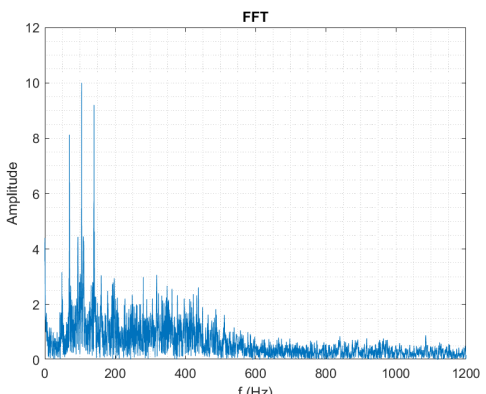

(a)

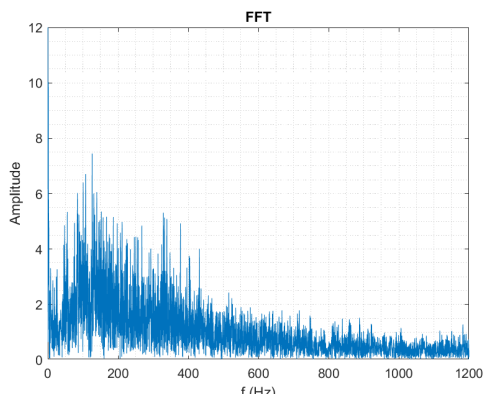

(b)

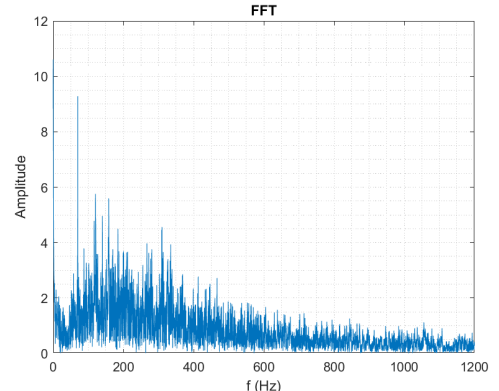

(c)

Figure 3. (a) Fast Fourier Transform (FFT) signal for Circle. (b) FFT signal for Square. (c) FFT signal for Triangle. 


\subsubsection{Envelope Time Signal}

We have decided to process the raw time signal, as the FFT signal's performance was worse than that of the raw time signal in the ANN training set. We have used the envelope MATLAB function that returns the upper and lower root-mean-square envelopes of the time signal. The envelope is determined using a sliding window of 200 samples, defined by the author. A sliding window greater than 200 samples results in a signal not generalized enough for the ANN. A sliding window smaller than 200 samples results in a signal which does not preserve the raw signal's original shape. As shown in Figure 4, the output of the envelope function is greater than or equal to zero, because it returns the root-mean-square envelope of the time signal. The 200 samples sliding window smooths the peaks and preserves the signal shape. Accordingly, it will be more general for the ANN to recognize the gesture, but we still have the problem of when the gesture started to be drawn. For this dataset, we had a success rate of $65 \%$.

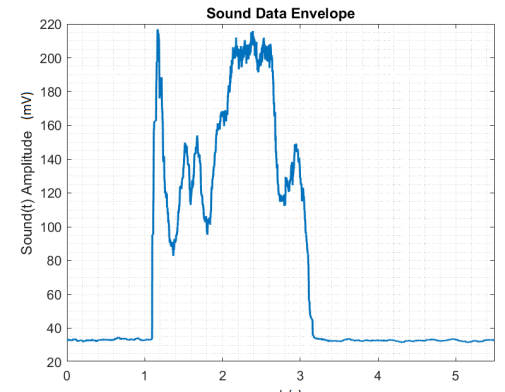

(a)

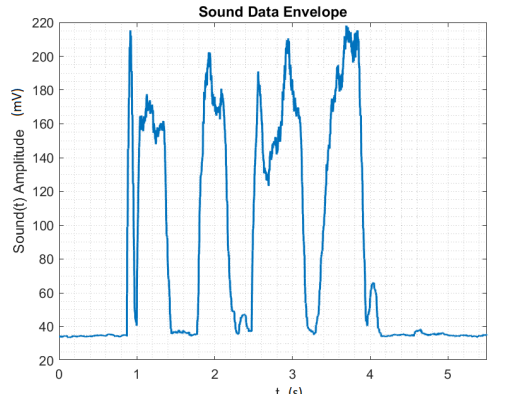

(b)

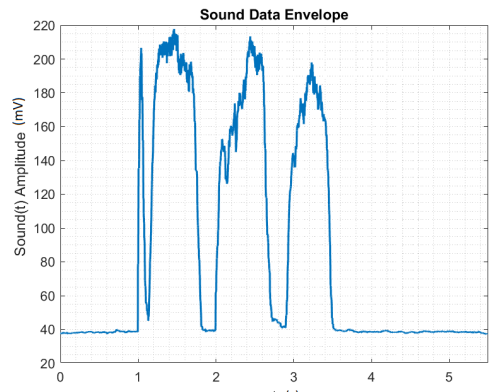

(c)

Figure 4. (a) Envelope time signal for the Circle. (b) Envelope time signal for the Square. (c) Envelope time signal for the Triangle.

\subsubsection{Time Scaled Envelope Signal}

Finally, we have time scaled the envelope signal employing the algorithm developed in this work and presented in Figure 5. In this data set, we first identify the initial touch, when considering that there is a peak every time the finger touches the surface, and we shift this data set to time 0 (zero). We identify the last touch, and we time scale the data set to the time interval [0,1], with 100 samples in between. Thus, the instant that the user begins to draw is no longer relevant. The second improvement was making the algorithm robust to the drawing speed, because users have different rhythms. We used time scaling to maintain the time scale of the data across different datasets.

Furthermore, all of the data sets were the same length of 100 samples. With the find peaks MATLAB function, and the options MinPeakHeight and MinPeakDistance, we could find peaks that had a minimum amplitude and ignored peaks that were very close to each other. Therefore, we have smoothed the peaks and preserved the initial shape of the signal. All of these improvements made it much easier for the ANN recognition task with the enhanced signals shown in Figure 6. Hence, for this dataset, we had the highest accuracy of $90 \%$ of success.

Table 1 presents the rate of success of each dataset evaluated to train the neural network. As explained before, the best result was with the time-scaled envelope signal, making it easier for the ANN recognition task.

Table 1. Success rate results for the different datasets that trained the ANN.

\begin{tabular}{cc}
\hline Signal & Success Rate \\
\hline Raw time signal & $33.00 \%$ \\
FFT signal & $15.00 \%$ \\
Envelope signal & $65.00 \%$ \\
Time scaled envelope signal & $90.00 \%$ \\
\hline
\end{tabular}




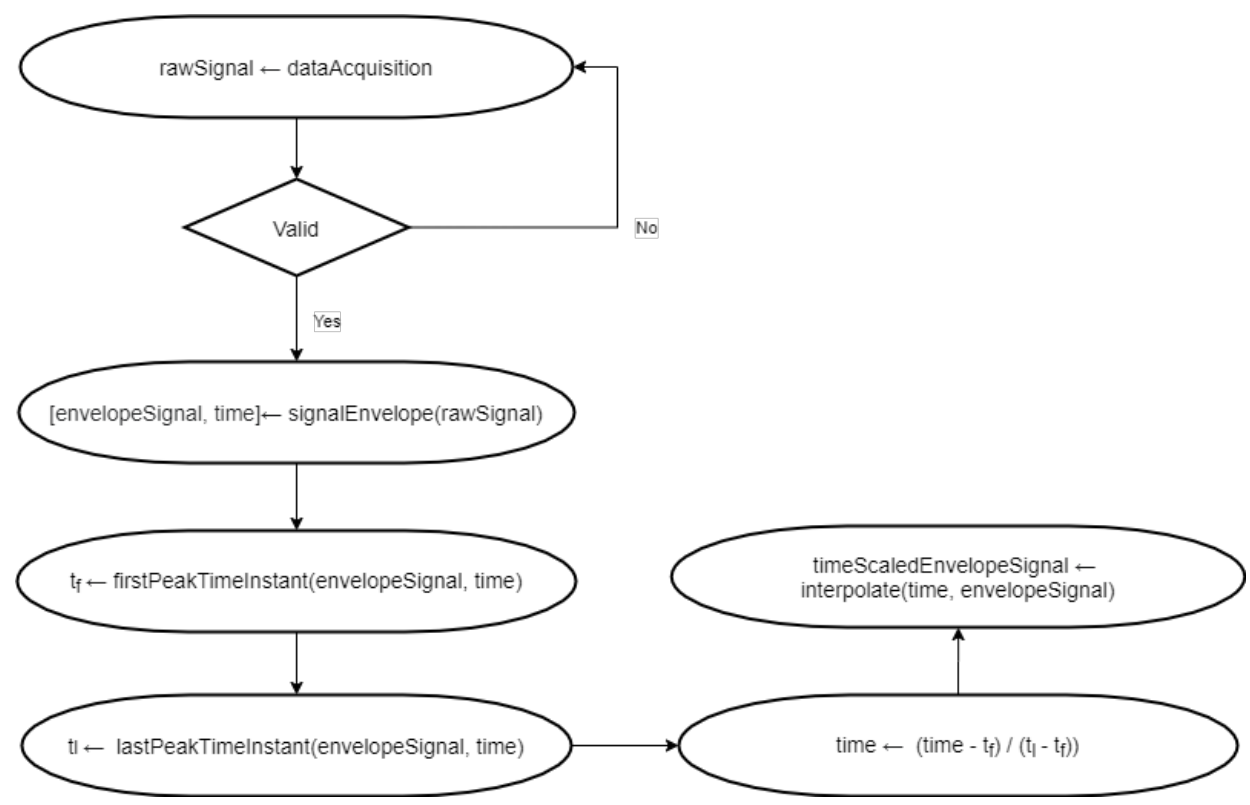

Figure 5. Proposed algorithm for processing the signal.

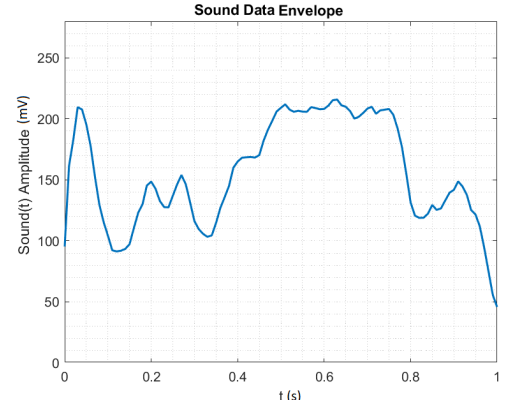

(a)

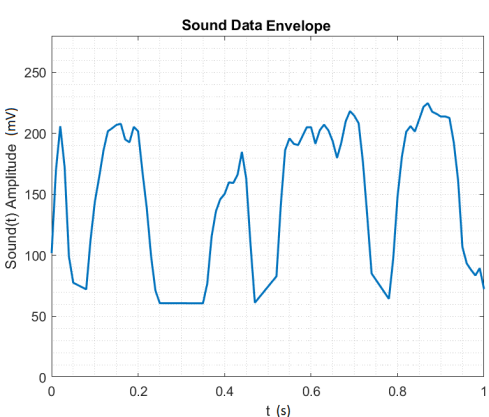

(b)

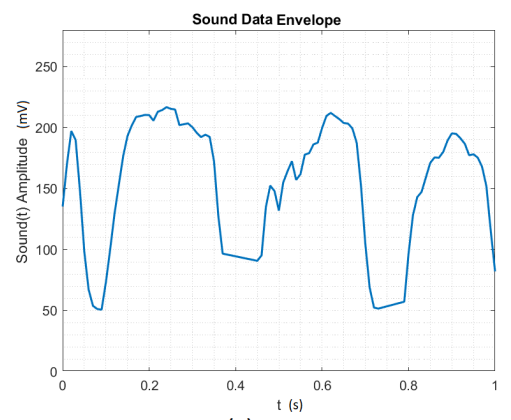

(c)

Figure 6. (a) Time scaled envelope signal for Circle. (b) Time scaled envelope signal for Square. (c) Time scaled envelope signal for Triangle.

From Figure 7, one can observe the development of the signal processing for the circle, from the raw signal in time until the time-scaled envelope signal that was evaluated by the algorithm proposed by the authors, as explained in Figure 5. We can see the improvements, as in Figure 7a, we have many more samples (more than 15,000) in the drawing, more peaks, and grooves, and the signal is not general enough for the ANN to have a high (above 85\%) recognition rate. In Figure $7 \mathrm{~b}$, we have a cleaner signal, with fewer samples and peaks, although we still cannot be general to realize when the drawing has started or the speed of the drawing. Finally, in Figure 7c, we observe the time-scaled envelope signal with only 100 samples. The proposed algorithm smooths the peaks and preserves the initial shape of the signal. 


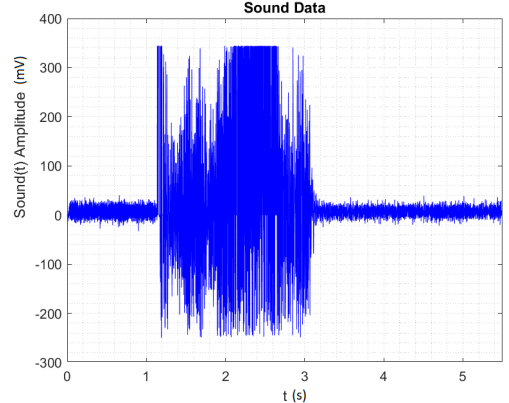

(a)

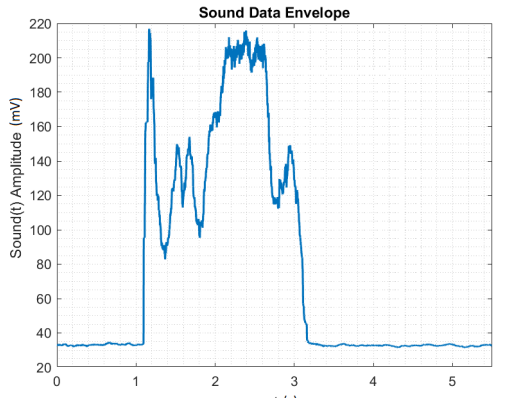

(b)

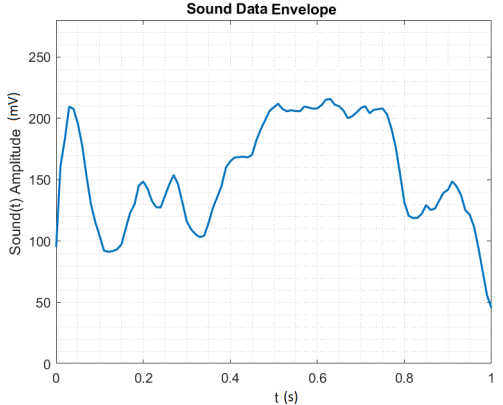

(c)

Figure 7. (a) Time signal for Circle. (b) Time envelope signal for Circle. (c) Time scaled envelope signal for Circle.

\subsection{Hidden Markov Models Experiment}

A stochastic approach for modeling an acoustic-based input is the Hidden Markov Model technique. A first-order Markov model is a finite set of states, where transitions between states are modeled by a transition probability matrix $A$, while assuming that the probability of being in state $S_{i}$ at time $t$ only depends on the state occupied at time $t-1$. If the state probability vector $\pi$ is known for $t=0$, the probability vector for the next observation moments can be recursively computed by [17]:

$$
\begin{gathered}
\pi_{t}=A \times \pi_{t-1} \\
\pi_{t}=A_{0}^{t}
\end{gathered}
$$

The state sequence of a Hidden Markov Model cannot be observed directly (hidden), and only the observation sequence is known. A Probability Density Function describes the probability of $P_{i}$ for the observation vector, given that the process is in a given state [17].

Over the past years, hidden Markov modeling has been one of the most popular and effective modeling techniques for acoustic time series. The HMMs are suitable for the classification from one or two-dimensional signals and it can be used when the information is incomplete or uncertain [18]. The reasons why this method has become so popular are the inherent (mathematically accurate) statistical structure; the facility and availability of training algorithms to estimate model parameters from finite sets of speech data training; the flexibility of the resulting recognition system in which you can easily change the size, type, or model architecture to suit words and sounds [19].

Taking into account that the acoustic speech signal has characteristics that are similar to the acoustic signal produced when a nail is dragged over a surface, previous works on speech recognition using HMMs were used as a basis and inspiration for this experiment. It is worth mentioning that the acoustic signal structure is similar, but the complexity of the signal dragged by a nail as compared to the speech signal is lower, since it is neither necessary to search for a range of phonemes nor to have a large database to train the model.

The choice of a topological configuration and the number of states in the model generally reflect the prior knowledge of the specific sound source to be modeled and is not related to mathematical treatability or implementation considerations [19]. The ideal number of states is best determined by executing experiments, because the relationship between the number of states and the HMM performance is very imprecise.

Luigi Rosa [20] proposed a fast and reliable algorithm for speech recognition based on Hidden Markov Models. The proposed approach uses frequency-related features robust to most types of noise.

The topological structure of the HMM implemented is left-right. These settings were pre-chosen by the toolbox of Luigi Rosa and his team [20] because they present good results in recognizing gestures on surfaces. We choose the left-right topology, because the gesture starts and ends in well-identified moments, and a sequential HMM well represents the sequential behavior of the movement. 
We developed an application in MATLAB to support gesture data collection and hidden Markov model training. For each gesture, the application prompts the user to start drawing, and then records the microphone signal for a three-second interval and saves it as a .wav extension by the audio write MATLAB function. Once recording completes, the system asks the user whether it should retain the particular drawing. After the application collects five drawings for a particular gesture, it repeats the same data collection process with the next gesture. After collecting data for all three gestures (circle, square, and triangle), the application generates a hidden Markov gesture model via the Luigi Rosa toolbox [20].

Figure 8 presents Luigi Rosa interface toolbox in MATLAB. We used the tab Add a new sound from files to train the model (it only accepts .wav file extensions).

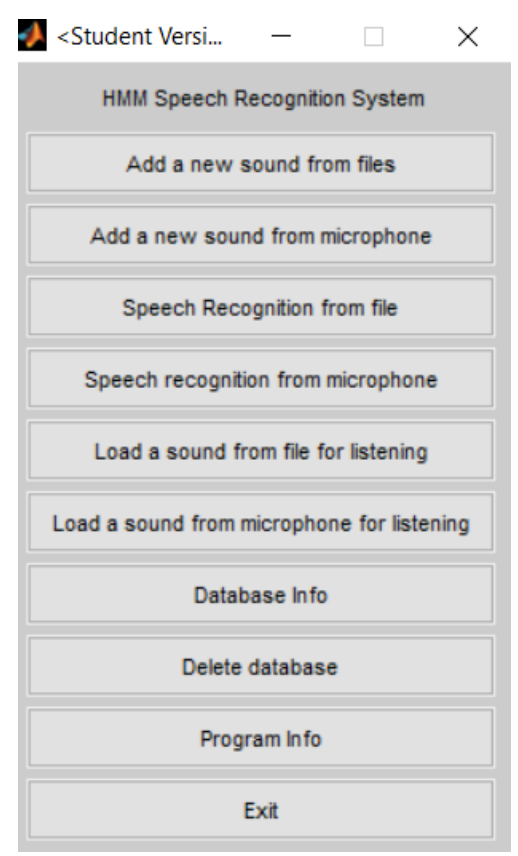

Figure 8. Luigi Rosa [20] interface toolbox in MATLAB.

The first dataset attempt to train the HMM was the raw time signal, such as for the ANN. We added five drawings for each gesture. Subsequently, using the tab Speech recognition from file in Luigi Rosa toolbox [20], we loaded a new sample to be recognized, and we obtained a recognition rate of $90 \%$.

\section{Discussion}

We address the research questions and the limitations of our proposed platform in this section when considering the literature review and the experimental results presented in this work.

\subsection{Addressing RQ1}

The RQ1 is widely addressed in the Acoustic sensing Related Works Section. Nevertheless, based on the related works presented, this section considers the tools that this work uses in more detail.

This paper has no intention of developing the toolboxes to train and build the neural network or the Markov model. Our focus is on the hardware and the embedded software development to acquire and process the signal and improve and test the datasets to feed the models. We used MATLAB's Neural Net Pattern Recognition Toolbox to train the network using scaled conjugate gradient backpropagation. In this toolbox, we can choose the network parameters, such as the number of neurons in the pattern recognition network's hidden layer, select samples for validation and testing, and it is possible to evaluate the ANN performance while using cross-entropy and confusion matrices. 
If the network's performance is not good enough, we can train it again, adjust the network size, or even import a larger data set to improve the network performance.

For the HMM, we have researched and found some toolboxes for speech recognition, such as the Georgia Tech Gesture Toolkit (GT2k) [21] and the gpdsHMM [18]. However, we had some issues in making them work, and we could not find support. Subsequently, we have tried the MATLAB toolbox implemented by the team of Luigi Rosa [20]. Accordingly, after collecting data for all of the gestures, the application generates a hidden Markov gesture model via Speech Recognition using Hidden Markov Models by Luigi Rosa [20]. We exchanged information with the Luigi Rosa team, and it was straightforward to make it work. Nevertheless, differently from the ANN toolbox, we have used, in this one, we can only supply the data to train the model, and we cannot choose any other parameter of the model. Therefore, the ANN toolbox is more configurable, gives the user more freedom to choose the parameters, and provides graphs that make it possible to evaluate the network's performance.

\subsection{Addressing $R Q 2$}

A central question when developing systems using machine learning is how much data are needed for training before accuracy levels off. In our work, we have chosen the target precision rate of at least $90 \%$, taking into account the precision rates from most of the related works: $89.5 \%$ from Scratch input [13], 90-95\% from Hambone [14], 95\% from Tapsense[12], 78.4\% from Pentelligence [9], 75\% from Stane [8], and 91.3\% from Ipanel [10]. We have tried the raw time signal and then the FFT signal for the ANN. The latter had the worst performance. We have then processed the raw time signal by taking the envelope and made some improvements such as interpolation to make the peaks and grooves smoother. After all these attempts, we have reached a success rate of $90 \%$, using for each gesture a ten drawings dataset, each composed of 100 samples, 1000 samples per gesture. Finally, for the HMM, we have achieved a success rate higher than $90 \%$ by training the model with the raw time signal and five drawings of each gesture, each drawing composed of 15,000 samples, which is, 75,000 samples per gesture.

Consequently, the choice of the dataset and the number of drawings for each machine learning technique is essential. For some drawings, the HMM has mistaken the square for the triangle and vice versa, but for the circle, we had a $100 \%$ successful recognition. It is essential to point out that HMM with a smaller number of drawings per gesture, shorter time to train, and smaller computational cost, then the ANN achieved the best precision rate.

\subsection{Addressing RQ3}

A previous work that inspired us was the Ipanel [10], because we have the same problems of tracking the acoustic signals that are generated by sliding fingers on a surface, and Ipanel's reached a robust performance against different levels of ambient noise and varying surface material. However, Ipanel [10] transformed the features into images and then employed the $\mathrm{CNN}$ to recognize the finger movement on the table. We have also tried to develop a CNN in this work, with MATLAB Deep Learning Toolbox. However, to train a network from scratch, the architect is required to define the number of layers and filters, along with other tunable parameters. Training an accurate model from scratch also requires massive amounts of data, on the order of millions of samples ([Online] The MathWorks, Inc, "Convolutional Neural Network" Available: Footnotes are not allowed in this journal, we moved it to here, please confirm. https:/ / www.mathworks.com/solutions/deep-learning/ convolutional-neural-network.html, accessed 28 December 2019).

Deep neural networks with a higher amount of training data and different topologies can achieve high recognition rates, as well as the impact of different surfaces or background noises [9]. Nevertheless, as we were looking for a simple and low computational cost solution, the CNN approach (or any deep neural network) turned out to be much more laborious, because of the need for this huge dataset and the longer time to train the network. 
Similarly, works, like TapSense [12], also inspired us. For the classification, they use the SVM technique provided in the Weka machine learning toolkit. However, as we can see in their works, training this classifier requires the collection of over 160 data points per gesture. Moreover, SVM also has some weakness when applied to tasks with low computational effort requirement, due to the large memory requirement and computation time of this training algorithm [22]. Consequently, the need for thousands of samples to train the model and the high computational effort would not fit our requirement of at most ten drawings per gesture and short training time (maximum of 2 min per gesture). These SVM limitations led us to try other techniques, such as HMM and ANN.

When considering the success rates for both machine learning techniques presented in Section 4, we achieved the highest success rate through the Markov model technique, which indicates that the proposed solution with HMM is simpler to implement and train than the ANN technique, and it is sufficient to solve the problem stated in the research questions.Therefore, HMM with a smaller dataset, shorter time to train, and computational cost lower than ANN achieved the best result.

\subsection{Discussion in How to Embed the HMM Trained into the Low-Cost Hardware}

In this section, we discuss how to generate code from the trained HMM automatically, cross-compile it, and then embed it into low-cost hardware. Thus, after training, the system would be independent of MATLAB software, which has a higher cost.

The first step is the installation of the hardware support package in Simulink. Second, develop the model, as shown in Figure 9. In this model, the Analog input acquires the raw signal. If the signal value is greater than 60, which means that someone is drawing on the surface, the Compare To Constant block applies a True value into the import $\operatorname{In} 1$ of the Enable Subsystem block, which has a memory block saving the raw signal during three seconds. The raw signal is then fed into the hidden Markov model previously generated through the MATLAB HMMModel function. Finally, the gesture recognition result (circle, triangle, or square) is printed on the serial output by the Serial Transmit block.

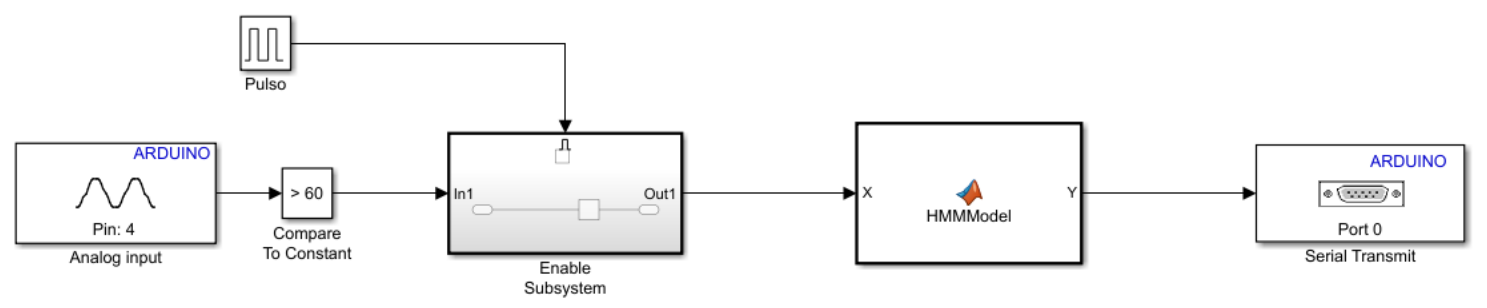

Figure 9. Model developed in Simulink to embedded on the hardware.

It is necessary to load, on the Arduino Nano, 25 MATLAB files, which exceed $140 \mathrm{~KB}$, with a specific input and output relationships, generated by Luigi Rosa's toolbox [20]. Hence, it is crucial to consider the memory specifications of the microcontroller that the developed HMM is to be embedded.

\subsection{Limitations}

We had some limitations on our platform, as we set our goal to use simple hardware and low computational cost for the machine learning algorithm. First, the maximum level of ambient noise had to be $45 \mathrm{~dB}$. We chose the aluminum surface to carry out the experiments because of its high speed of sound propagation, and the use of stethoscopes to acquire sounds was crucial, because it provides a high level of suppression of environmental noise. Second, we used a simple initial alphabet of geometrical figures: circle, square, and triangle.

We found that small variations in sensor placement caused significant variations in signal characteristics. Thus, repeatable sensor placement is critical for practical inter-session training and recognition. 


\section{Conclusions}

In this paper, we presented the design of a low-cost acoustic sensing gesture recognition system based on surface scratch input. In the context of the design, we validated the robustness of the HMM technique to recognizing gestures on surfaces and with low computational costs concerning time and physical memory use. We obtained at least a $90 \%$ success rate with five drawings per gesture, and a short training time, a maximum of two minutes per gesture.

Besides the design of the system, we focused on processing the dataset that trains the models to achieve better performance. To do so, we implemented two machine learning techniques, namely ANN and HMM, to support answering the research questions. The solution proposed with the HMM classifier is simpler to implement and train than the ANN technique and it is sufficient to solve the problem. For the training stage, with five drawings per gesture and the raw time signal, the HMM achieved a success rate of $90 \%$. Therefore, the HMM with a smaller number of drawings, shorter signal processing time, and training time obtained the best result. The results also demonstrated the importance of data representation for the efficient processing of sensory information for the ANNs.

In future work, we are investigating the use of multiple microphones for the physical sensing setup providing an extension of using location and improve the classification precision. Additionally, a hardware implementation, e.g., FPGA (Field Programmable Gate Array), can be considered.

Author Contributions: Conceptualization, B.S.M.; Formal analysis, A.P. and S.O.D.L.; Investigation, B.S.M., A.P. and S.O.D.L.; Methodology, A.P. and S.O.D.L.; Project administration, A.P. and S.O.D.L.; Software, B.S.M.; Supervision, A.P. and S.O.D.L.; Writing-original draft, B.S.M.; Writing-review \& editing, A.P., S.O.D.L. and B.S.M. All authors have read and agreed to the published version of the manuscript.

Funding: This research was funded by CNPq (Brazilian National Research Council) grant.

Acknowledgments: The authors would like to thank the CNPq for the financial support and everyone from the LIEC (Electronic Instrumentation and Control Laboratory UFCG) and Embedded (Embedded Systems and Pervasive Computing Laboratory) who supported the development of this work. We also thank COPELE (Graduate Course in Electrical Engineering), Federal University of Campina Grande, Brazil.

Conflicts of Interest: The authors declare no conflict of interest.

\section{References}

1. Lopes, P.; Jota, R.; Jorge, J.A. Augmenting touch interaction through acoustic sensing. In Proceedings of the ACM International Conference on Interactive Tabletops and Surfaces 2011, Kobe, Japan, 13-16 November 2011; ACM: New York, NY, USA, 2011; pp. 53-56.

2. Yin, H.; Zhou, A.; Liu, L.; Wang, N.; Ma, H. Ubiquitous Writer: Robust Text Input for Small Mobile Devices via Acoustic Sensing. IEEE Int. Things J. 2019, 6, 5285-5296. [CrossRef]

3. Luo, G.; Yang, P.; Chen, M.; Li, P. HCI on the Table: Robust Gesture Recognition Using Acoustic Sensing in Your Hand. IEEE Access 2020, 8, 31481-31498. [CrossRef]

4. Alonso-Martín, F.; Gamboa-Montero, J.J.; Castillo, J.C.; Castro-González, Á.; Salichs, M.Á. Detecting and Classifying Human Touches in a Social Robot Through Acoustic Sensing and Machine Learning. Sensors 2017, 17, 1138. [CrossRef] [PubMed]

5. Chou, T.-R.; Lo, J.-C. Research on tangible acoustic interface and its applications. In Proceedings of the 2nd International Conference on Computer Science and Electronics Engineering, Hangzhou, China, 22-23 March 2013; Atlantis Press: Amsterdam, The Netherlands, 2013.

6. Soldado, P.M.G. Toolkit for Gesture Classification through Acoustic Sensing. Master's Thesis, Técnico Lisboa, Lisboa, Portugal, 2015.

7. Rekimoto, J. Smartskin: An infrastructure for freehand manipulation on interactive surfaces. In Proceedings of the SIGCHI Conference on Human Factors in Computing Systems, Minneapolis, MN, USA, 20-25 April 2002; ACM: New York, NY, USA, 2002; pp. 113-120.

8. Murray-Smith, R.; Williamson, J.; Hughes, S.; Quaade, T. Stane: Synthesized surfaces for tactile input. In Proceedings of the SIGCHI Conference on Human Factors in Computing Systems, Florence, Italy, 5-10 April 2008; ACM: New York, NY, USA, 2008; pp. 1299-1302. 
9. Schrapel, M.; Stadler, M.-L.; Rohs, M. Pentelligence: Combining pen tip motion and writing sounds for handwritten digit recognition. In Proceedings of the 2018 CHI Conference on Human Factors in Computing Systems, Montreal, QC, Canada, 21-26 April 2018; ACM: New York, NY, USA, 2018; pp. 1-11.

10. Chen, M.; Yang, P.; Xiong, J.; Zhang, M.; Lee, Y.; Xiang, C.; Tian, C. Your table can be an input panel: Acoustic-based device-free interaction recognition. Proc. ACM Interact. Mob. Wearable Ubiquitous Technol. 2019, 3, 1-21. [CrossRef]

11. Harrison, C.; Tan, D.; Morris, D.; Starner, T. Skinput: Appropriating the body as an input surface. In Proceedings of the SIGCHI Conference on Human Factors in Computing Systems, Atlanta, GA, USA, 10-15 April 2010; ACM: New York, NY, USA, 2010; pp. 453-462.

12. Harrison, C.; Schwarz, J.; Hudson, S.E. Tapsense: Enhancing finger interaction on touch surfaces. In Proceedings of the 24th Annual ACM Symposium on User Interface Software and Technology, Santa Barbara, CA, USA, 16-19 October 2011; ACM: New York, NY, USA, 2011; pp. 627-636.

13. Harrison, C.; Hudson, S.E. Scratch input: Creating large, inexpensive, unpowered and mobile finger input surfaces. In Proceedings of the 21st Annual ACM Symposium on User Interface Software and Technology, Monterey, CA, USA, 19-22 October 2008; ACM: New York, NY, USA, 2008; pp. 205-208.

14. Deyle, T.; Palinko, S.; Poole, E.S.; Starner, T. Hambone: A bio-acoustic gesture interface. In Proceedings of the 2007 11th IEEE International Symposium on Wearable Computers, Boston, MA, USA, 11-13 October 2007; pp. 3-10.

15. Braun, A.; Krepp, S.; Kuijper, A. Acoustic tracking of hand activities on surfaces. In Proceedings of the 2nd International Workshop on Sensor-Based Activity Recognition and Interaction, Rostock, Germany, 24-25 June 2015; ACM: New York, NY, USA, 2015; pp. 1-5.

16. Vujicic, T.; Matijevic, T.; Ljucovic, J.; Balota, A.; Sevarac, Z. Comparative analysis of methods for determining number of hidden neurons in artificial neural network. In Proceedings of the 27th Central European Conference on Information and Intelligent Systems (CECIIS 2016), Varazdin, Croatia, 21-23 September 2016; Faculty of Organization and Informatics Varazdin: Varaždin, Croatia, 2016; pp. 219-223.

17. Price, J.; Eydgahi, A. Design of MATLAB-based automatic speaker recognition systems. In Proceedings of the 9th International Conference on Engineering Education-T4J-1, San Juan, Puerto Rico, 23-28 July 2006.

18. David, S.; Ferrer, M.; Travieso, C.; Alonso, J.B. gpdsHMM: A hidden Markov model toolbox in the MATLAB environment. In Proceedings of the Complex Systems, Intelligence and Modern Technological Applications Conference, Cherbourg, France, 19-22 September 2004; pp. 476-479.

19. Juang, B.H.; Rabiner, L.R. Hidden markov models for speech recognition. Technometrics 1991, 33, $251-272$. [CrossRef]

20. Rosa, L. Speech Recognition Using Hidden Markov Models. Available online: http://www. advancedsourcecode.com/hmmspeech.asp (accessed on 3 December 2019).

21. Westeyn, T.; Brashear, H.; Atrash, A.; Starner, T. Georgia tech gesture toolkit: Supporting experiments in gesture recognition. In Proceedings of the International Conference on Multimodal Interfaces, Vancouver, BC, Canada, 5-7 November 2003; ACM: New York, NY, USA, 2003; pp. 85-92.

22. Lopes, C.; Perdigão, F. Event detection by HMM, SVM and ANN: A comparative study. In Proceedings of the International Conference on Computational Processing of the Portuguese Language, Canela, Brazil, 24-26 September 2018; Springer: Aveiro, Portugal, 2008; pp. 1-10.

(C) 2020 by the authors. Licensee MDPI, Basel, Switzerland. This article is an open access article distributed under the terms and conditions of the Creative Commons Attribution (CC BY) license (http://creativecommons.org/licenses/by/4.0/). 